\title{
CORRELATES OF FAMILY CONFLICT IN FAMILIES RAISING CHILDREN WITH DIABETES
}

\author{
J. Pascoe, P. Breyer, C. Mcnicholas, M. Lee \\ Pediatrics, Wright State University, Dayton, OH, USA
}

Background and aims: Conflicts within families of children with diabetes may have adverse effects on their disease management. This study aims to identify factors related to family conflict in families with diabetic children.

Methods: Cross-sectional survey of 262 birth parents recruited from a children's hospital Diabetes Clinic between January 2010 and January 2011. The survey included demographics, the Social Capital Scale (SCS), Diabetes Family Conflict Scale (DFCS), Center for Epidemiologic Studies Depression Scale (CES-D), and Pediatric Quality of Life Scale (PedsQL).

Results: 221 were birth mothers and 41 were birth fathers. $71 \%$ were married, $58 \%$ were employed fulltime, and $59 \%$ reported more than a high school education. $84 \%$ of the children were white, and $49 \%$ were girls. Mean age (years) was 11.9 (SD, 3.9). Total DFCS was significantly correlated with SCS ( $\mathrm{r}=-0.17, \mathrm{p}<$ 0.01), CES-D ( $r=0.27, \mathrm{p}<0.001)$, and PedsQL $(\mathrm{r}=-0.45, \mathrm{p}<0.001)$. There were no differences for families with sons or daughters in DFCS $(p=0.57)$, SCS $(p=0.83)$, CES-D $(p=0.24)$, and PedsQL $(p=0.76)$. There was a significant pattern of factors related to DFCS among families raising daughters, mothers reported higher DFCS than fathers $(26.7\{0.8\}$ vs. $22.6\{0.8\}, \mathrm{p}<0.05)$. Parents reported significantly higher DFCS in families with adolescent daughters (age $>12$ years) compared to younger daughters $(27.4\{0.9\}$ vs. $24.9\{1.0\}, \mathrm{p}<$ $0.01)$.

Conclusions: The CES-D , PedsQL and SCS were all significantly correlated with the DFCS. For daughters of all ages, mothers reported more conflict than fathers. Parents of adolescent daughters reported more conflict than parents of younger daughters. 